\title{
In Search of Scrub Typhus: A Prospective Analysis of Clinical and Epidemiological Profile of Patients from a Tertiary Care Hospital in New Delhi
}

\author{
Nitin Gupta1, Rama Chaudhry', Sushil K. Kabra², Rakesh Lodha², Bijay R. Mirdha1, \\ Bimal K. Das ${ }^{1}$, Naveet Wig' ${ }^{3}$ Vishnubhatla Sreenivas ${ }^{4}$ \\ ${ }^{1}$ Department of Microbiology, All India Institute of Medical Sciences, New Delhi, India \\ ${ }^{2}$ Department of Paediatrics, All India Institute of Medical Sciences, New Delhi, India \\ ${ }^{3}$ Department of Medicine, All India Institute of Medical Sciences, New Delhi, India \\ ${ }^{4}$ Department of Biostatistics, All India Institute of Medical Sciences, New Delhi, India \\ Email:drramach@gmail.com
}

Received 19 October 2015; accepted 20 November 2015; published 23 November 2015

Copyright (C) 2015 by authors and Scientific Research Publishing Inc.

This work is licensed under the Creative Commons Attribution International License (CC BY). http://creativecommons.org/licenses/by/4.0/

\section{Open Access}

\section{Abstract}

Introduction: Scrub typhus is a febrile illness caused by Orientia tsutsugamushi which is transmitted by the larval stage of trombiculid mites. It has varied manifestations ranging from mild disease to fatal illness. The classical manifestations include fever, rash, lymphadenopathy and eschar. Serology is the preferred diagnostic tool with indirect immunofluorescence assay (IFA) as the current gold standard. Material \& Methods: A total of 229 clinically suspected cases of Scrub typhus from $15^{\text {th }}$ of October 2013 to $1^{\text {th }}$ of October 2015 were enrolled in the study. Clinical, epidemiological and laboratory data of the patients were collected and analysed. The method for diagnosis was IgM immunofluorescence assay. Results: A total of 33 cases $(14.4 \%)$ of scrub typhus were detected. Most of the cases (87\%) presented in the months of September to November. Fever of $>39^{\circ} \mathrm{C}$ was seen in all 33 cases. Rash and eschar were seen in $24.2 \%$ and $18.2 \%$ cases, respectively. Lymphadenopathy (18.2\%), hepatomegaly $(48.5 \%)$, splenomegaly $(21.2 \%)$ and icterus $(15.2 \%)$ were seen in some cases. Pulmonary manifestations were noted in $69.7 \%$ of cases. Acute kidney injury (18.2\%), encephalitis $(21.2 \%)$, and myocarditis (15.15\%) were the other complications observed. Common haematological abnormalities noted in patients of scrub typhus were anaemia $(45.6 \%)$, leucocytosis $(45.6 \%)$, thrombocytopenia $(48.5 \%)$ and transaminitis $(54.5 \%) .10$ cases were treated by azithromycin while doxycycline was used for treatment in 28 cases. Conclusion: The nonspecific symptoms and lack of classical manifestations make the diagnosis of scrub typhus difficult. There is a definite need to increase awareness and heighten the suspicion, especially in the light of increasing number of patients presenting with atypical manifestations. 


\section{Keywords}

\section{Scrub Typhus, India, Epidemiology}

\section{Introduction}

Scrub typhus is caused by an obligate intracellular bacterium, Orientia tsutsugamushi. This bacterium has been excluded taxonomically from the genus Rickettsia based on the 16S rRNA gene sequences [1]. Scrub typhus is still clinically grouped under rickettsioses along with Rickettsia spp., Ehlrichia spp., Anaplasma spp. and Coxiella burnetii [2]. It is transmitted by the larval stage of trombiculid mites, commonly known as chiggers [3]. Infected chiggers are found particularly in areas of heavy scrub vegetation and scrub typhus is typically assumed to be associated with forest region. Though, cases from urban settings are now beginning to emerge.

Around a million cases of scrub typhus are reported each year with an estimated mortality of around $6 \%$ in untreated patients [3] [4]. Scrub typhus is predominantly reported from an area commonly known as the "Tsutsugamushi triangle" that extends from east of Russia and Japan in the north to Australia in the south and Afghanistan in the west. Scrub typhus is widely reported from most Asian countries. In 1999, the World Health Organization (WHO) stated, "Scrub typhus is probably one of the most under diagnosed and underreported febrile illnesses requiring hospitalization in the region". Scrub typhus has known to occur in India since long. Even though the first case was reported in Assam in 1930s, scrub typhus has remained as a neglected disease in most parts of the country [5].

Up until now, Scrub typhus was assumed to be prevalent only in the Himalayan belt (Himachal Pradesh, Jammu \& Kashmir) and southern peninsula (Tamil Nadu, Kerala), but there has been an increase in the number of cases reported from various states including Bihar, West Bengal, Rajasthan, Maharashtra, Karnataka and north eastern states [5]-[8]. The central part of India including Delhi, Haryana and Uttar Pradesh has apparently remained elusive of the ongoing epidemic [9] [10]. Scrub typhus has varied manifestations ranging from a mild disease to a fatal illness. The early classical manifestations include fever, rash, lymphadenopathy and eschar. The primary papular lesion formed at the site of chigger bite enlarges up to $1 \mathrm{~cm}$, undergoes central necrosis and forms a black eschar with a surrounding erythematous halo. Progression of severe scrub typhus may manifest as acute respiratory distress, meningoencephalitis and acute renal failure [11]. Serology is the preferred diagnostic tool with indirect immunofluorescence assay (IFA) as the current gold standard [12]. The objective of the study was to determine the prevalence of scrub typhus in New Delhi and analyse the clinical and epidemiological profile of patients diagnosed with scrub typhus.

\section{Material \& Methods}

A total of 229 clinically suspected cases of Scrub typhus who were either admitted or attended the out-patient department (OPD) in the All India Institute of Medical Sciences (AIIMS), New Delhi, from $15^{\text {th }}$ of October 2013 to $15^{\text {th }}$ of October 2015 were enrolled in the study. The hospital is an apex care centre catering to patients from all over India but most patients come from the neighbouring states of Uttar Pradesh, Haryana, Madhya Pradesh and Bihar. A suspected case of Scrub typhus was defined as undifferentiated fever with or without any of the following manifestations: eschar, rash, headache, lymphadenopathy and multi-organ involvement.

Clinical, epidemiological and laboratory data of the cases were collected and analysed using a structured questionnaire. For diagnosis of Scrub typhus, blood sample $(2 \mathrm{ml})$ was collected during the acute phase and the serum was separated. Suspected cases were serologically evaluated using gold standard for diagnosis-IgM Immunofluorescence assay (Fuller Laboratories, USA). The IFA slides in this kit utilized 4 strains (Karp, Kato, Gilliam and Boryong) which were propagated in L292 cells. The diluted patient sera were incubated in the wells of the slide to enable reaction between antibody present in the serum and antigen on the slide. A fluorescence conjugate was used to label the antigen-antibody complexes which were then visualised under fluorescent microscope. A positive reaction was seen as fluorescent rod forms in the background of counterstained red cells.

Data analysis: The prevalence of scrub typhus among the patients presenting with undifferentiated febrile illness and the epidemiological and clinical features of patients diagnosed with scrub typhus were expressed as percentage. The $95 \%$ confidence intervals for the prevalence of scrub typhus was calculated. The data was ana- 
lysed with Stata version 12.1.

\section{Results}

During the study period, there were 229 patients who were clinically suspected of scrub typhus. A total of 33 cases $(14.4 \%$, 95\% CI 10.1\% - 19.6\%) of scrub typhus were detected (14 in 2013, 11 in 2014, 8 in 2015) using IgM IFA (Figure 1). The patients resided in different states of North and Central India, however majority of our cases were from Delhi (21/33, 63.6\%) and Haryana (6/33, 18.2\%) (Figure 2). Majority of the cases (87\%) presented in the months of September to November (87.9\%, 29/33) (Figure 3). Male patients (18/33, 54.6\%) were

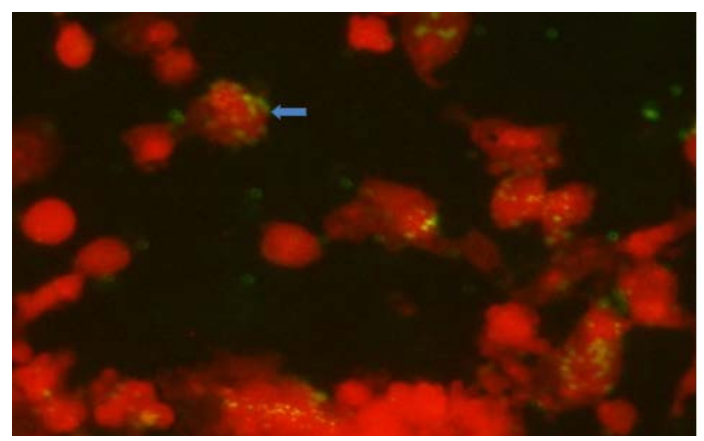

Figure 1. Immunofluorescence assay with the arrow showing fluorescent antigen antibody complexes in red background.

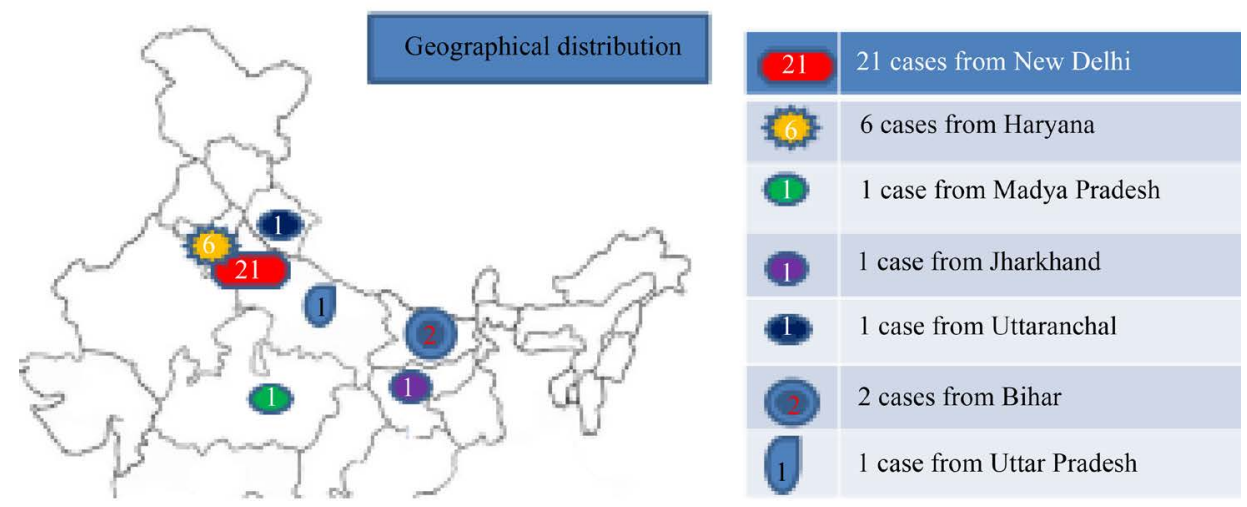

Figure 2. Geographical distribution of Scrub typhus in North India.

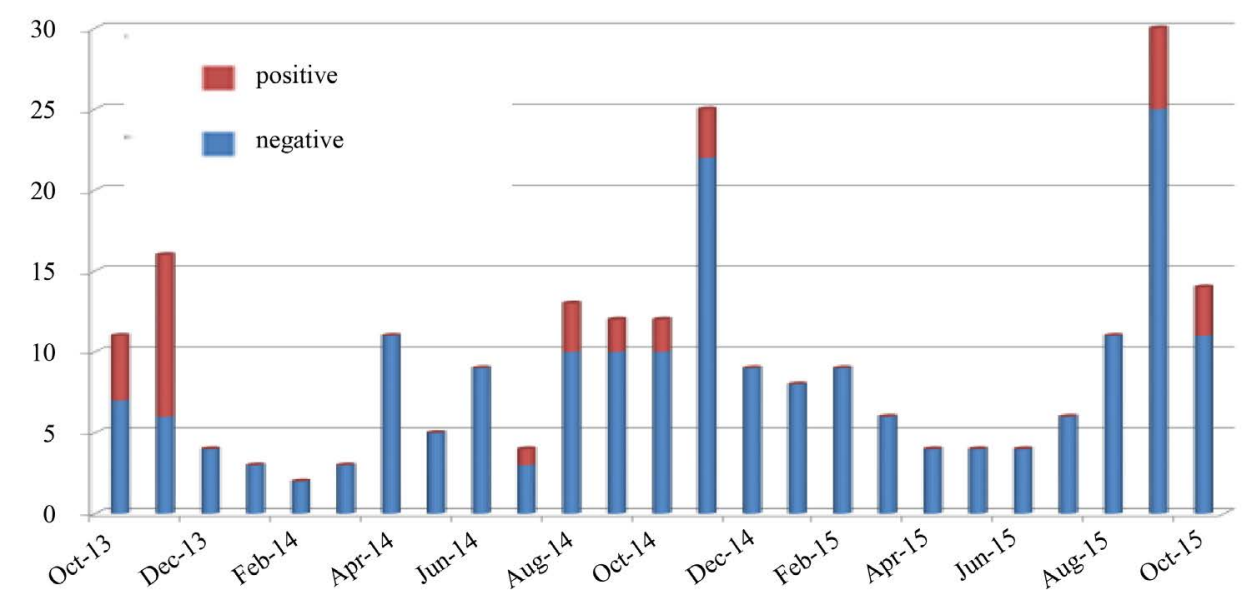

Figure 3. Seasonal distribution of Scrub typhus. 
almost in same numbers as that of female patients (15/33, 45.4\%). The age of the patients with diagnosed scrub typhus ranged from 4 to 69 years. There were only 6 cases of paediatric age group. Most number of cases (16/33, 48.4\%) were in the age group of 10 - 30 years (Table 1). A recent visit to forest or its vicinity was found in only $18.2 \%(6 / 33)$ cases. Fever of $>39^{\circ} \mathrm{C}$ was seen in all 33 cases. The duration of fever at the time of diagnosis ranged from 4 - 20 days with mean duration of 12 days (Table 2). Rash was only seen in 24.2\% (8/33) cases. The rash was mostly maculopapular and was predominantly found on the trunk (Table 2). Eschar was only seen in 18.2\% (6/33) cases with chest and extremities being the most common location (Figure 4) (Table 2). Lymphadenopathy $(18.2 \%, 6 / 33)$, hepatomegaly $(48.5 \%, 16 / 33)$ and splenomegaly $(21.2 \%, 7 / 33)$ were also reported in some of the cases. Visible icterus was seen in only $15.2 \%$ (5/33) cases. Pulmonary manifestations were noted in $69.7 \%$ cases with most cases presenting with dyspnoea (54.5\%) (Table 2). Around 18.2\% (6/33) cases progressed to Acute Respiratory Distress syndrome (ARDS). Acute kidney injury evidenced by increased urea and creatinine was seen in $18.2 \%$ (6/33) cases. Features of encephalitis were seen in $21.2 \%$ cases (7/33) (Table 2). Myocarditis was seen in 15.15\% (5/33) of cases while a single case of pericardial effusion was observed. Common haematological abnormalities noted in patients of scrub typhus were anaemia (45.6\%, 15/33), leucocytosis $(45.6 \%, 15 / 33)$, thrombocytopenia $(48.5 \%, 16 / 33)$ and transaminitis $(54.5 \%, 18 / 33)$. Pancytopenia was noted in $15.2 \%$ (5/33) cases (Table 2). 10 cases were treated by azithromycin while doxycycline was used for treatment in 28 cases. 5 cases were treated with both doxycycline and azithromycin. All the cases had a favourable outcome.

\section{Discussion}

In our prospective observational study conducted over 24 months, 33 cases (14.4\%) were diagnosed with scrub typhus. A recent study conducted in New Delhi reported the seropositivity for scrub typhus in suspected patients to be around $16.05 \%$ [9]. Other studies conducted in different parts of India reported prevalence rates ranging from $30.8 \%$ to $46 \%$ [6] [13]-[15]. Scrub typhus is a seasonal disease with high incidence in the post monsoon season, probably because of the spurt in the growth of scrubs creating a favourable environment for the mite populations. All the diagnosed patients presented in the months of July to November. 87\% of these cases presented in the months of September to November. Similar observations have been reported in other studies [14] [16]. This seasonal pattern should alert the treating physicians to consider scrub typhus in the list of differential that commonly includes only dengue and malaria during these months.

The target cell for Scrub typhus is monocytes, endothelium and cardiac myocytes while the major pathology is vasculitis. This leads to micro-vascular leakage which in turn leads to edema, hypo-perfusion and ischemic injury [17]. The clinical features of scrub typhus are nonspecific and require a high degree of suspicion. The classical manifestations like rash, lymphadenopathy and eschar are not commonly reported. This creates a lot of confusion and a heavy reliance on these features to clinically diagnose scrub typhus has been discouraged. Rash is uncommonly seen in scrub typhus as was also observed in our study (24.2\%). It usually appears on the $3^{\text {rd }}$ to $5^{\text {th }}$ day of fever. It starts with a macule and later on gets converted to maculopapular, petechial or haemorrhagic rash [18]. Eschar if seen is pathognomic of Scrub typhus as the other differential for eschar are Rickettsial pox and Anthrax which are either not reported from India or do not fit these clinical features. Although, presence of eschar is indicative of scrub typhus, the reverse is not true. In our study, out of 223 cases of suspected scrub typhus without eschar, $10.3 \%$ of the patients were diagnosed with scrub typhus. Eschar has been found to be

Table 1. Age distribution of patients diagnosed with scrub typhus.

\begin{tabular}{ccc} 
Age (in years) & Frequency $(\mathbf{n}=\mathbf{3 3})$ \\
\hline $0-10$ & 6 \\
$11-20$ & 10 \\
$21-30$ & 6 \\
$31-40$ & 5 \\
$41-50$ & 3 \\
$51-60$ & 1 \\
$>60$ & 2 \\
\hline
\end{tabular}


Table 2. Presenting features of scrub typhus.

\begin{tabular}{|c|c|c|c|}
\hline & & Presenting feature & Frequency $(\mathrm{n}=33)$ \\
\hline \multirow[t]{5}{*}{ A } & & Fever & 33 \\
\hline & 1 & Fever for $<7$ days & 12 \\
\hline & 2 & Fever for 7 - 12 days & 12 \\
\hline & 3 & Fever for 13 - 18 days & 6 \\
\hline & 4 & Fever for $>18$ days & 3 \\
\hline \multirow[t]{8}{*}{ B } & & Rash & 8 \\
\hline & 1 & Rash location—diffuse & 2 \\
\hline & 2 & Rash location—-trunk & 3 \\
\hline & 3 & Rash location—face & 2 \\
\hline & 4 & Rash location-extremity & 1 \\
\hline & 1 & Rash type-maculopapular & 6 \\
\hline & 2 & Rash type-petechial & 1 \\
\hline & 3 & Rash type-haemorrhagic & 1 \\
\hline \multirow[t]{5}{*}{$\mathrm{C}$} & & Eschar & 6 \\
\hline & 1 & Eschar site-Chest & 2 \\
\hline & 2 & Eschar site—Arm & 2 \\
\hline & 3 & Eschar site—Back & 1 \\
\hline & 4 & Eschar site—Leg & 1 \\
\hline \multirow[t]{5}{*}{$\mathrm{D}$} & & Lymphadenopathy & 6 \\
\hline & 1 & Lymphadenopathy site—Cervical \& axillary & 3 \\
\hline & 2 & Lymphadenopathy site-Cervical only & 1 \\
\hline & 3 & Lymphadenopathy site—-Inguinal & 1 \\
\hline & 4 & Lymphadenopathy site_-Generalised & 1 \\
\hline $\mathrm{E}$ & & Icterus & 5 \\
\hline \multirow[t]{4}{*}{$\mathrm{F}$} & & Pulmonary manifestations & 23 \\
\hline & 1 & Dyspnoea & 18 \\
\hline & 2 & Chest pain & 2 \\
\hline & 3 & Cough & 3 \\
\hline \multirow[t]{6}{*}{ G } & & Chest X-ray findings & 13 \\
\hline & 1 & Bilateral perihilar opacities & 8 \\
\hline & 2 & Bilateral pleural effusion & 1 \\
\hline & 3 & Pulmonary edema & 2 \\
\hline & 4 & Unilateral pleural effusion & 1 \\
\hline & 5 & Consolidation & 1 \\
\hline $\mathrm{H}$ & & Hepatomegaly & 16 \\
\hline I & & Splenomegaly & 7 \\
\hline $\mathrm{J}$ & & Encephalitis & 7 \\
\hline I & & Seizures & 2 \\
\hline K & & Myocarditis & 5 \\
\hline $\mathrm{L}$ & & Pericardial effusion & 1 \\
\hline M & & Anaemia & 15 \\
\hline $\mathrm{N}$ & & Leucocytosis & 15 \\
\hline $\mathrm{O}$ & & Thrombocytopenia & 16 \\
\hline $\mathrm{P}$ & & Transaminitis & 18 \\
\hline
\end{tabular}




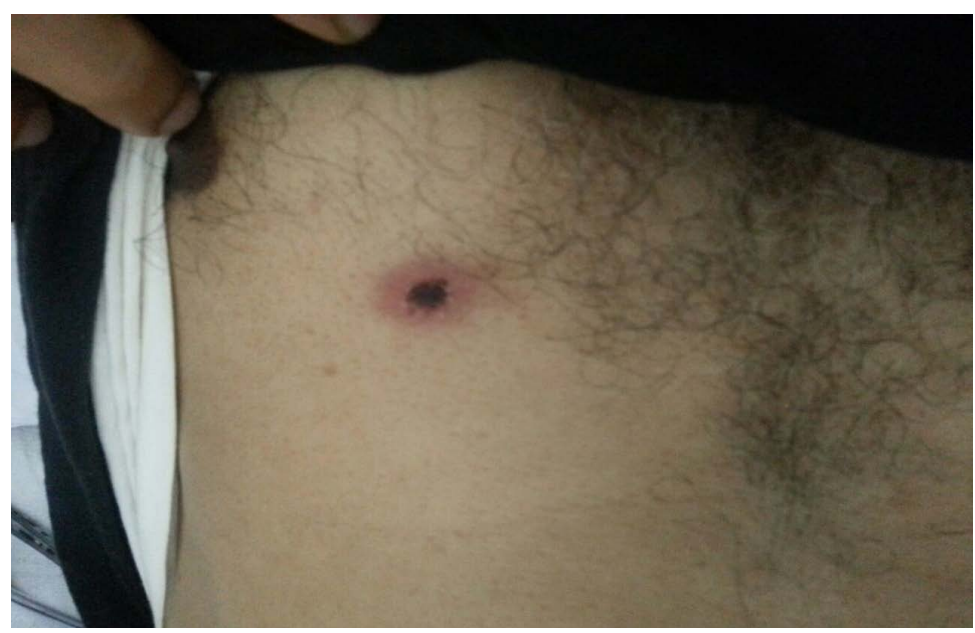

Figure 4. Eschar on the chest of a person diagnosed with Scrub typhus.

uncommon (9.5\% - 45\%) in most studies from South East Asia and Indian subcontinent which was in concordance with our findings (18.2\%) [13] [16] [19]. An eschar is usually found on the areas with tight clothing and commonly includes axillae, groin, chest and abdomen [5]. The clandestine location of the eschar may be the reason that it is frequently missed on inspection and explains the low prevalence. Also, it is difficult to visualize on dark skinned individuals. In our study eschar was commonly found on the chest and the upper extremities. Lymphadenopathy was observed in $18.2 \%$ of cases in the present study.

Hepatomegaly and splenomegaly were seen in $48.5 \%$ and $21.2 \%$ of cases, respectively. Both hepatomegaly and splenomegaly in the same patient was seen in $15.2 \%$ of cases. Other studies have also recorded high incidence of hepatosplenomegaly. The presence of splenomegaly serves as an important sign to distinguish scrub typhus from dengue where splenomegaly is seldom seen. One of the striking observation in our study was the high preponderance of pulmonary manifestations in the form of dyspnoea and cough with some cases (18.2\%) getting complicated by the development of ARDS. ARDS is a life threating complication but the pathology behind it in scrub typhus is still unclear. It is understood that there is widespread alveolar damage without any evidence of vasculitis [20]. Recent studies from India show an increase in the incidence of ARDS to around $8 \%-10 \%$ in cases of Scrub typhus [6] [16]. Acute Kidney Injury (AKI) in scrub typhus is mostly mild and non-oliguric. Renal recovery is seen in almost all the cases. The incidence of renal failure ranges from $18 \%$ to $66.4 \%$ [6] [21] [22]. AKI in our study was noticed in $18 \%$ of cases. Scrub typhus can affect both the central and peripheral nervous system. Most common CNS complication is meningoencephalitis which is due to vasculitis caused by the organism. CSF studies reveal mild pleocytosis with lymphocyte predominance and increased protein levels. The incidence of encephalitis seen in our study was $21 \%$. Additionally seizures were seen in two of these cases but none of the patient showed any focal neurological signs or any signs of meningeal irritation. The typical CSF findings were noted in only two cases. The incidence of meningoencephalitis in other Indian studies ranged from 9.5\% to 23.3\% [6] [16] [22]. Even though cardiac myocyte is a known target of scrub typhus, very few cases of myocarditis in scrub typhus has been reported in literature [4]. We reported 5 cases of myocarditis in our study. High percentage of mild hepatitis as evidenced by the increase in liver enzymes has been noted in other studies like ours (54.5\%) [6] [7] [22] [23]. A small percentage of these cases manifest as icterus. Leucocytosis (45.6\%) and thrombocytopenia (48.5\%) were commonly found in patients of scrub typhus in other studies as well [7] [22]. Thrombocytopenia in scrub typhus is usually not accompanied with an elevated hematocrit which helps to differentiate it from dengue.

Doxycycline has been considered as a drug of choice for treatment of scrub typhus [24]. The recommended duration of treatment is 7 days but it can be extended up to 15 days in complicated cases [5]. Azithromycin has shown to have comparable efficacy when compared to doxycycline but the resolution of symptoms has shown to be faster in doxycycline compared to azithromycin [25] [26]. Rifampicin can be used in combination with azithromycin or doxycycline in cases of poor response to doxycycline alone [27]. Treatment of scrub typhus was initiated with doxycycline in most cases (84.8\%). All 33 of the scrub typhus patients recovered completely and were discharged. 
The limitation of our study was that strain variation in relation to virulence and severity was not taken into account. Also, because ours is an apex institute, most patients are referred from lower centres and our data may not be representative of the general population.

\section{Conclusion}

Scrub typhus is an acute febrile illness associated with life-threatening complications, if not diagnosed and treated on time. Due to the nonspecific symptoms and lack of classical manifestations in most cases, diagnosis is difficult. Also the co-endemicity and similar seasonal patterns of other vector borne diseases like malaria and dengue lead to more confusion. There is a definite need to increase awareness and heighten the suspicion, especially in the light of increasing number of patients presenting with atypical manifestations.

\section{References}

[1] Ohashi, N., et al. (1995) Phylogenetic Position of Rickettsia Tsutsugamushi and the Relationship among Its Antigenic Variants by Analyses of 16S rRNA Gene Sequences. FEMS Microbiology Letters, 125, 299-304. http://dx.doi.org/10.1111/j.1574-6968.1995.tb07372.x

[2] Dumler, J.S., et al. (2001) Reorganization of Genera in the Families Rickettsiaceae and Anaplasmataceae in the Order Rickettsiales: Unification of Some Species of Ehrlichia with Anaplasma, Cowdria with Ehrlichia and Ehrlichia with Neorickettsia, Descriptions of Six New Species Combinations and Designation of Ehrlichia Equi and "HGE Agent” as Subjective Synonyms of Ehrlichia Phagocytophila. International Journal of Systematic and Evolutionary Microbiology, 51, 2145-2165. http://dx.doi.org/10.1099/00207713-51-6-2145

[3] Paris, D.H., et al. (2013) Unresolved Problems Related to Scrub Typhus: A Seriously Neglected Life-Threatening Disease. American Journal of Tropical Medicine and Hygiene, 89, 301-307. http://dx.doi.org/10.4269/ajtmh.13-0064

[4] Taylor, A.J., Paris, D.H. and Newton, P.N. (2015) A Systematic Review of Mortality from Untreated Scrub Typhus (Orientia tsutsugamushi). PLoS Neglected Tropical Diseases, 9, e0003971. http://dx.doi.org/10.1371/journal.pntd.0003971

[5] Rahi, M., et al. (2015) DHR-ICMR Guidelines for Diagnosis \& Management of Rickettsial Diseases in India. Indian Journal of Medical Research, 141, 417-422. http://dx.doi.org/10.4103/0971-5916.159279

[6] Mahajan, S.K., et al. (2006) Scrub Typhus in Himalayas. Emerging Infectious Diseases, 12, 1590-1592. http://dx.doi.org/10.3201/eid1210.051697

[7] Chrispal, A., et al. (2010) Scrub Typhus: An Unrecognized Threat in South India-Clinical Profile and Predictors of Mortality. Tropical Doctor, 40, 129-133. http://dx.doi.org/10.1258/td.2010.090452

[8] Sharma, R., et al. (2014) Analysis of Two Outbreaks of Scrub Typhus in Rajasthan: A Clinico-Epidemiological Study. Journal of the Association of Physicians of India, 62, 24-29.

[9] Mittal, V., et al. (2012) Serological Evidence of Rickettsial Infections in Delhi. Indian Journal of Medical Research, 135, 538-541.

[10] Chaudhry, D., et al. (2009) Rickettsial Diseases in Haryana: Not an Uncommon Entity. Journal of the Association of Physicians of India, 57, 334-337.

[11] Peter, J.V., Sudarsan, T.I., Prakash, J.A. and Varghese, G.M. (2015) Severe Scrub Typhus Infection: Clinical Features, Diagnostic Challenges and Management. World Journal of Critical Care Medicine, 4, 244-250.

[12] Blacksell, S.D., Bryant, N.J., Paris, D.H., Doust, J.A., Sakoda, Y. and Day, N.P.J. (2007) Scrub Typhus Serologic Testing with the Indirect Immunofluorescence Method as a Diagnostic Gold Standard: A Lack of Consensus Leads to a Lot of Confusion. Clinical Infectious Diseases, 44, 391-401. http://dx.doi.org/10.1086/510585

[13] Sharma, A., Mahajan, S., Gupta, M.L., Kanga, A. and Sharma, V. (2005) Investigation of an Outbreak of Scrub Typhus in the Himalayan Region of India. Japanese Journal of Infectious Diseases, 58, 208-210.

[14] Kamarasu, K., Malathi, M., Rajagopal, V., Subramani, K., Jagadeeshramasamy, D. and Mathai, E. (2007) Serological Evidence for Wide Distribution of Spotted Fevers \& Typhus Fever in Tamil Nadu. The Indian Journal of Medical Research, 126, 128-130.

[15] Gurung, S., Pradhan, J. and Bhutia, P.Y. (2013) Outbreak of Scrub Typhus in the North East Himalayan Region-Sikkim: An Emerging Threat. Indian Journal of Medical Microbiology, 31, 72-74. http://dx.doi.org/10.4103/0255-0857.108729

[16] Vivekanandan, M., Mani, A., Priya, Y.S., Singh, A.P., Jayakumar, S. and Purty, S. (2010) Outbreak of Scrub Typhus in Pondicherry. The Journal of the Association of Physicians of India, 58, 24-28.

[17] Rathi, N. and Rathi, A. (2010) Rickettsial Infections: Indian Perspective. Indian Pediatrics, 47, 157-164. 
http://dx.doi.org/10.1007/s13312-010-0024-3

[18] Mahajan, S.K. (2005) Scrub Typhus. Journal of the Association of Physicians of India, 53, 954-958.

[19] Varghese, G.M., Abraham, O.C., Mathai, D., Thomas, K., Aaron, R., Kavitha, M.L. and Mathai, E. (2006) Scrub Typhus among Hospitalised Patients with Febrile Illness in South India: Magnitude and Clinical Predictors. Journal of Infection, 52, 56-60. http://dx.doi.org/10.1016/j.jinf.2005.02.001

[20] Park, J.S., Jee, Y.K., Lee, K.Y., Kim, K.Y., Myong, N.H. and Seo, P.W. (2000) Acute Respiratory Distress Syndrome Associated with Scrub Typhus: Diffuse Alveolar Damage without Pulmonary Vasculitis. Journal of Korean Medical Science, 15, 343-345. http://dx.doi.org/10.3346/jkms.2000.15.3.343

[21] Attur, R.P., Kuppasamy, S., Bairy, M., Nagaraju, S.P., Pammidi, N.R., Kamath, V., et al. (2013) Acute Kidney Injury in Scrub Typhus. Clinical and Experimental Nephrology, 17, 725-729. http://dx.doi.org/10.1007/s10157-012-0753-9

[22] Varghese, G.M., Trowbridge, P., Janardhanan, J., Thomas, K., Peter, J.V., Mathews, P., Abraham, O.C. and Kavitha, M.L. (2014) Clinical Profile and Improving Mortality Trend of Scrub Typhus in South India. International Journal of Infectious Diseases, 23, 39-43. http://dx.doi.org/10.1016/j.ijid.2014.02.009

[23] Mathai, E., Rolain, J.M., Verghese, G.M., Abraham, O.C., Mathai, D., Mathai, M. and Raoult, D. (2003) Outbreak of Scrub Typhus in Southern India during the Cooler Months. Annals of the New York Academy of Sciences, 990, 359-364. http://dx.doi.org/10.1111/j.1749-6632.2003.tb07391.x

[24] Sheehy, T.W., Hazlett, D. and Turk, R.E. (1973) Scrub Typhus. A Comparison of Chloramphenicol and Tetracycline in Its Treatment. Archives of Internal Medicine, 132, 77-80. http://dx.doi.org/10.1001/archinte.1973.03650070069010

[25] Phimda, K., Hoontrakul, S., Suttinont, C., Chareonwat, S., Losuwanaluk, K., Chueasuwanchai, S., et al. (2007) Doxycycline versus Azithromycin for Treatment of Leptospirosis and Scrub Typhus. Antimicrobial Agents and Chemotherapy, 51, 3259-3263. http://dx.doi.org/10.1128/AAC.00508-07

[26] Fang, Y., Huang, Z., Tu, C., Zhang, L., Ye, D. and Zhu, B.P. (2012) Meta-Analysis of Drug Treatment for Scrub Typhus in Asia. Internal Medicine, 51, 2313-2320. http://dx.doi.org/10.2169/internalmedicine.51.7816

[27] Watt, G., Chouriyagune, C., Ruangweerayud, R., Watcharapichat, P., Phulsuksombati, D., Jongsakul, K., et al. (1996) Scrub Typhus Infections Poorly Responsive to Antibiotics in Northern Thailand. Lancet, 348, 86-89. http://dx.doi.org/10.1016/S0140-6736(96)02501-9 